\title{
Comment améliorer la prise en charge des patients âgés aux urgences ?
}

\author{
How to Improve Medical Care of Elderly Patients in our Emergency Department?
}

\author{
P. Ray $\cdot$ J. Boddaert \\ (C) SFMU et Lavoisier SAS 2016
}

Avec plus de 14 millions de passages aux urgences en France par an (2004), les services d'urgence sont une des principales voies d'accès à l'hôpital pour de nombreux patients, dont les patients âgés (PA). Les patients âgés représentent approximativement 10 à $20 \%$ de tous les passages aux urgences [1] et $50 \%$ des patients hospitalisés au décours du passage au service d'urgence (SU). Or la prise en charge des PA est difficile pour un certain nombre de raisons. D'abord, ces patients sont caractérisés par leur vulnérabilité. Ensuite, ils cumulent pathologies et traitements (5 à 7 environ), qui favorisent le risque de pathologie iatrogène. Enfin, le contexte même de l'urgence rend cette prise en charge encore plus difficile car le temps manque pour reconstituer antécédents, traitements habituels, anamnèse et examen clinique exhaustif. Des travaux montrent que le temps d'attente des PA est plus long [2] et que malheureusement ce délai de prise en charge augmenté est associé à une morbimortalité plus importante [3].

Améliorer la prise en charge en urgence des PA représente donc un enjeu important, et repose sur des principes relativement « simples » sur le papier : faire attendre le moins possible « inutilement » des PA particulièrement vulnérables, évaluer au mieux la complexité de ces patients, appréhender le risque iatrogène des traitements et des explorations invasives et associer au plus tôt une expertise gériatrique, en faisant intervenir les gériatres aux urgences ou grâce à la mise en place de filières dédiées originales.

Dans ce numéro des AFMU, l'équipe des urgences de 1'hôpital Amboise Paré à Boulogne-Billancourt rapporte leur

P. Ray $(\bowtie)$

Service d'accueil des urgences, hôpital Tenon, AP-HP,

4 rue de la Chine, F-75020 Paris, UPMC-Paris

6, DHU FAST

e-mail : patrick.ray@tnn.aphp.fr

J. Boddaert

Département de gériatrie, unité péri-opératoire de gériatrie, groupe hospitalier Pitié Salpêtrière, AP-HP,

47-83 boulevard de l'Hôpital, F-75013 Paris,

UPMC-Paris 6, DHU FAST expérience de mise en place d'un « circuit court » dédié aux PA. Dans cette étude de type avant-après, le fast-track consiste en l'admission des PA à l'unité d'hospitalisation de courte durée (UHCD) dans les deux heures, afin de diminuer leur temps de passage en SU [4]. Ce type de parcours accéléré nécessite une disponibilité quasi continue des lits en unité d'observation, une collaboration avec les gériatres pour cette prise en charge, et une organisation interne des urgentistes et du SU en général. Compte tenu de la méthodologie de l'étude, les auteurs ne peuvent conclure sur le service rendu aux PA, en particulier sur une diminution des complications hospitalières, de la durée moyenne de séjour, de la mortalité, sur le risque d'admettre inutilement des patients qui auraient dû rentrer à domicile, ou encore sur le retentissement sur les autres patients consultants en SAU. Mais, les auteurs démontrent une bonne adhésion au protocole par les médecins urgentistes, ce qui suggère une sensibilisation des urgentistes à cette problématique, ce qui est déjà une première étape essentielle. Dans les Hauts de Seine, la prévalence des PA est importante, comme le montre la proportion de PA aux urgences de l'hôpital Ambroise Paré. Cette filière originale répond aussi à une organisation interne (urgences médicales et chirurgicales séparées) de la structure d'urgence et rend cette filière d'ores et déjà attrayante, puisque, par exemple, les urgentistes « chirurgie/trauma » n'ont pas ou peu à s'occuper du versant médical de PA consultants pour chute compliquée d'un traumatisme.

Reste à montrer son impact clinique. D'autres filières dédiées aux urgentistes ont vu le jour en France récemment. La filière centrée autour de l'unité péri-opératoire de gériatrie (UPOG) mise en place à la Pitié-Salpêtrière et dédiée essentiellement aux patients avec fracture de l'extrémité supérieure du fémur a montré une amélioration de la morbimortalité par rapport au schéma classique de l'hospitalisation des PA en orthopédie [5]. D'autres filières sont intéressantes, comme la filière d'urgence dédiée aux PA ouverte à Limoges (Tchalla A et al., données personnelles). Si l'évaluation scientifique de toutes ces initiatives est une étape indispensable, il ne faut pas négliger l'environnement institutionnel. En effet, la diffusion d'une organisation efficace est une dernière étape difficile, car les contraintes locales 
propres à chaque hôpital sont des éléments clefs dans l'implémentation de nouvelles bonnes pratiques. Ainsi, l'ouverture d'un département d'aval des urgences n'est pas forcément adaptée à tel hôpital et à tel SU, et de même l'ouverture d'un circuit court dépend des moyens alloués et de la typologie des patients qui consultent.

Restent que ces initiatives innovantes visant à améliorer nos procédures représentent une démarche que tous les SU doivent adopter, selon les problématiques qu'ils rencontrent. Et le vieillissement de la population concerne tous les SU.

Liens d'intérêts : Les auteurs déclarent ne pas avoir de lien d'intérêt.

\section{Références}

1. Aminzadeh F, Dalziel WB (2002) Older adults in the emergency department: a systematic review of patterns of use, adverse outcomes, and effectiveness of interventions. Ann Emerg Med 39:238-47

2. Freund Y, Vincent-Cassy C, Bloom B, et al (2013) Association between age older than 75 years and exceeded target waiting times in the emergency department: a multicenter cross-sectional survey in the Paris metropolitan area, France. Ann Emerg Med 62:449-56

3. Richardson D (2006) Increase in patient mortality at 10 days associated with emergency department overcrowding. Med J Aust 184:213-6

4. Arrouy L, Strohmenger L, Attal J, et al (2016) Évaluation de la mise en place d'un fast-track pour les personnes âgées 1 de plus de 85 ans dans un service d'urgence. Ann Fr Med Urgence 6:233-9

5. Boddaert J, Cohen-Bittan J, Khiami F, et al (2014) Postoperative admission to a dedicated geriatric unit decreases mortality in elderly patients with hip fracture. PLoS One 9:e83795 